\title{
Heterochirality and Halogenation Control Phe-Phe Hierarchical Assembly
}

\author{
Slavko Kralj, Ottavia Bellotto, Evelina Parisi, Ana M. Garcia, Daniel Iglesias, Sabrina Semeraro, \\ Caterina Deganutti, Paola D’Andrea, Attilio V. Vargiu, Silvano Geremia, Rita De Zorzi,* \\ and Silvia Marchesan*
}

Cite This: ACS Nano 2020, 14, 16951-16961

ACCESS | Lill Metrics \& More | 回 Article Recommendations | st Supporting Information

ABSTRACT: Diphenylalanine is an amyloidogenic building block that can form a versatile array of supramolecular materials. Its shortcomings, however, include the uncontrolled hierarchical assembly into microtubes of heterogeneous size distribution and well-known cytotoxicity. This study rationalized heterochirality as a successful strategy to address both of these pitfalls and it provided an unprotected heterochiral dipeptide that self-organized into a homogeneous and optically clear hydrogel with excellent ability to sustain fibroblast cell proliferation and viability. Substitution of one L-amino acid with its D-enantiomer preserved

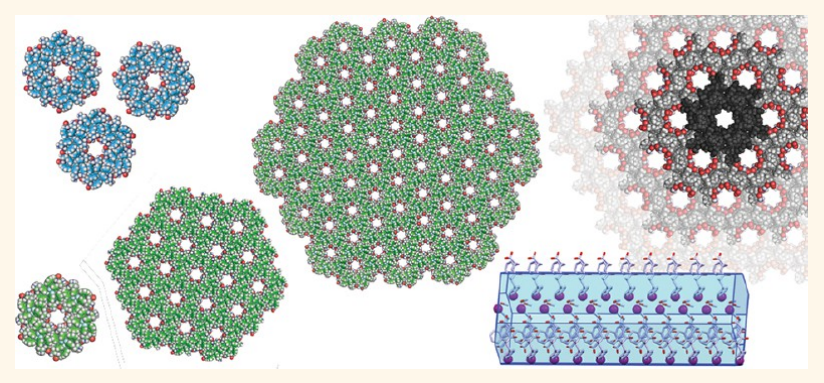
the ability of the dipeptide to self-organize into nanotubes, as shown by single-crystal XRD analysis, whereby the pattern of electrostatic and hydrogen bonding interactions of the backbone was unaltered. The effect of heterochirality was manifested in subtle changes in the positioning of the aromatic side chains, which resulted in weaker intermolecular interactions between nanotubes. As a result, D-Phe-L-Phe self-organized into homogeneous nanofibrils with a diameter of $4 \mathrm{~nm}$, corresponding to two layers of peptides around a water channel, and yielded a transparent hydrogel. In contrast with homochiral Phe-Phe stereoisomer, it formed stable hydrogels thermoreversibly. D-Phe-L-Phe displayed no amyloid toxicity in cell cultures with fibroblast cells proliferating in high numbers and viability on this biomaterial, marking it as a preferred substrate over tissue-culture plastic. Halogenation also enabled the tailoring of D-Phe-L-Phe self-organization. Fluorination allowed analogous supramolecular packing as confirmed by XRD, thus nanotube formation, and gave intermediate levels of bundling. In contrast, iodination was the most effective strategy to augment the stability of the resulting hydrogel, although at the expense of optical transparency and biocompatibility. Interestingly, iodine presence hindered the supramolecular packing into nanotubes, resulting instead into amphipathic layers of stacked peptides without the occurrence of halogen bonding. By unravelling fine details to control these materials at the meso- and macro-scale, this study significantly advanced our understanding of these systems.

KEYWORDS: peptides, chirality, self-assembly, D-amino acids, hydrogels, phenylalanine, halogenation

$\mathrm{P}$ eptide self-assembly has attracted great interest over the last two decades as a facile strategy to achieve supramolecular smart materials. ${ }^{1}$ Peptides are convenient building blocks thanks to their chemical diversity, wellestablished chemistry, biodegradability, and possibility to

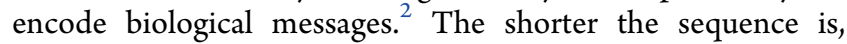
the lower the production costs are, and the easier the synthesis and purification can be. It is thus not surprising that minimalistic self-assembling motifs composed of two to three amino acids have become popular targets of investigation. ${ }^{3}$ Their self-assembly in environmentally benign solvents, such as water, is known to be favored by the use of aromatic $\mathrm{N}$-caps, among which the most popular are fluorenylmethyloxycarbonyl or Fmoc, and naphthalene or Nap derivatives. ${ }^{4,5}$ However, the resulting lower solubility may render their handling more challenging and may also require organic solvents. Additionally, concerns exist over the biological fate of such $\mathrm{N}$-caps, thus the search is active for substitutes. ${ }^{6-9}$ Uncapped, short peptides are attractive alternatives. Unfortunately, their selfassembly and gelation are far more challenging to predict, as exemplified by a seminal work by Tuttle and Ulijn, which scored the hydrophobicity of all 8,000 combinations of $\mathrm{L}$ -

Received: July 20, 2020

Accepted: October 30, 2020

Published: November 11, 2020

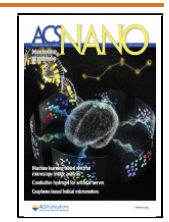


amino acids in tripeptides in search of gelators yet it described only 4 gelling sequences. ${ }^{10} \mathrm{~A}$ clear relationship between chemical structure and gelation propensity is very challenging to find, as reported also by Hauser and co-workers on a large tripeptide library. ${ }^{11}$

Phenylalanine (Phe) stands out among the 20 natural amino acids for its unmatched propensity toward self-assembly, and indeed it was reported to form toxic fibrils on its own at high concentrations. ${ }^{12}$ Phe-Phe is possibly the most versatile selfassembling motif in minimalistic gelators, and subtle chemical variations led to a variety of nanomorphologies. ${ }^{13-17}$ Studies continue to emerge describing its useful properties, ranging from piezoelectricity to ferroelectricity, for applications that span printing to semiconductors. ${ }^{18-22}$ At the base of these features are the electronic properties that arise from the peculiar supramolecular arrangement of Phe-Phe in nanotubes. $^{23}$ Clearly, there is ample scope to tailor derivatives of this compound toward the development of cost-effective supramolecular materials.

In particular, halogenation was reported to favor selforganization and hydrogelation of Fmoc-Phe derivatives. ${ }^{24,25}$ Iodination can be advantageous through the establishment of halogen-bonding, as reported in a pentapeptide. ${ }^{26}$ However, the design of halogenated peptides that self-organize into supramolecular hydrogels is not trivial, since the formation of macroscopic and stable hydrogels is the result of a fine balance between many steric and electronic factors. Phe-Phe gave rise to metastable hydrogels that underwent syneresis over time, ${ }^{27,28}$ and $\mathrm{N}$-terminal halogenation was shown to affect self-assembly, so that the poorly water-soluble L-(4-I)-Phe-LPhe and L-(4-I)-Phe-L-(4-I)-Phe were reported to form crystals. $^{29}$

Inspired by these works, we chose diphenylalanine (1) as a model compound and studied the self-organization of heterochiral D-Phe-L-Phe (2), as well as its halogenated derivatives D-(2-F)-Phe-L-Phe (3), D-(3-F)-Phe-L-Phe (4), D(4-F)-Phe-L-Phe (5), and D-(4-I)-Phe-L-Phe (6), as shown in Chart 1 . Halogenation of the $\mathrm{C}$-terminus was not included as it was previously shown not to affect the self-assembly of the parent amyloid sequence Lys-Leu-Val-Phe-Phe. ${ }^{30}$ Iodination in the ortho- or meta-positions of Phe was not studied as it was envisaged to likely disrupt assembly due to iodine's bulky nature and ability to engage in halogen bonding with carbonyl groups. ${ }^{30}$ Our choice of heterochiral sequences was dictated by the observation that introducing D-amino acids at selected positions has proven successful as a strategy to achieve hydrogels from uncapped tripeptides. ${ }^{31,32}$ In this manner, it is possible to orient favorably the side chains of hydrophobic sequences to access amphiphilic gelling structures, as was recently reviewed. ${ }^{33}$ Heterochirality is gaining momentum as a strategy to tailor peptide self-assembly. ${ }^{34-36}$ Uncapped dipeptides are very attractive building blocks due to their chemical simplicity. Furthermore, enantiomers are expected to display the same self-assembly behavior in achiral environments, therefore, investigation of compounds 2-6 will shed light also on the self-assembly of their mirror-image L-D dipeptide isomers.

\section{RESULTS AND DISCUSSION}

Peptide Self-Assembly into Nanostructured Hydrogels. Each compound shown in Chart 1 was synthesized and purified by reverse-phase HPLC (see Supporting Information, Sections S1-S6 for spectroscopic characterization), which
Chart 1. Phe-Phe Compounds Studied for Self-Assembly<smiles>NC(Cc1ccccc1)C(=O)N[C@@H](Cc1ccccc1)C(=O)O</smiles><smiles>NC(Cc1ccccc1)C(=O)NC(Cc1ccccc1)C(=O)O</smiles><smiles>N[C@@H](Cc1ccccc1F)C(=O)N[C@@H](Cc1ccccc1)C(=O)O</smiles><smiles>NC(Cc1cccc(F)c1)C(=O)NC(Cc1ccccc1)C(=O)O</smiles><smiles>N[C@@H](Cc1ccc(F)cc1)C(=O)N[C@@H](Cc1ccccc1)C(=O)O</smiles><smiles>N[C@@H](Cc1ccc(I)cc1)C(=O)N[C@@H](Cc1ccccc1)C(=O)O</smiles>

revealed increasing retention times from $\mathbf{1}$ to $\mathbf{6}$, with only minor differences among fluorinated regioisomers (Table 1 ).

Table 1. Hydrophobicity and Minimum Gelling Concentration (mgc) of Dipeptides 1-6

\begin{tabular}{cccc} 
compound & HPLC Rt $(\mathrm{min})$ & $\log \mathrm{P}^{41}$ & $\operatorname{mgc}(\mathrm{mM})$ \\
$\mathbf{1}$ & 6.0 & $2.3 \pm 0.5$ & 20 \\
$\mathbf{2}$ & 7.1 & $2.3 \pm 0.5$ & 20 \\
$\mathbf{3}$ & 7.1 & $2.4 \pm 0.5$ & 15 \\
$\mathbf{4}$ & 7.2 & $2.4 \pm 0.5$ & 10 \\
$\mathbf{5}$ & 7.3 & $2.4 \pm 0.5$ & 7 \\
6 & 7.9 & $3.6 \pm 0.5$ & 4 \\
\hline
\end{tabular}

All compounds were dissolved in aqueous sodium phosphate at $\mathrm{pH} \sim 12$, since at alkaline $\mathrm{pH}$ the repulsion between the negative charges of the peptide anions impedes self-assembly. Subsequent dilution with an equal volume of mildly acidic phosphate buffer led to a final $\mathrm{pH}$ of 7.3. Under these conditions, peptide zwitterions formed salt bridges between their charged termini, triggering self-assembly. All compounds 1-6 formed supramolecular hydrogels as confirmed by oscillatory rheology (see Supporting Information, Section $\mathrm{S} 8$ ). The minimum gelling concentration $(\mathrm{mgc})$ was inversely proportional to the HPLC retention time, which is an experimental measure of hydrophobicity, ${ }^{37}$ as shown in Table 1. However, not all hydrogels were stable over time (Figure 1). Compounds 1 and 4 displayed syneresis, as already reported for $1 .^{27,28}$ This feature, which consists of the gel contraction with concomitant release of liquid, may be attractive for the capture and release of small molecules, ${ }^{38,39}$ such as pollutants or drugs, provided that release kinetics are controlled. Compounds 2, 3, and 6 yielded stable and homogeneous hydrogels; in particular, compounds 2 and 3 formed materials of high transparency, which is a desirable feature for optical applications. Compound $\mathbf{5}$ crystallized over days, suggesting a low energetic barrier and high structural similarity between the two states, gel and crystal. ${ }^{40}$ Hydrogel 


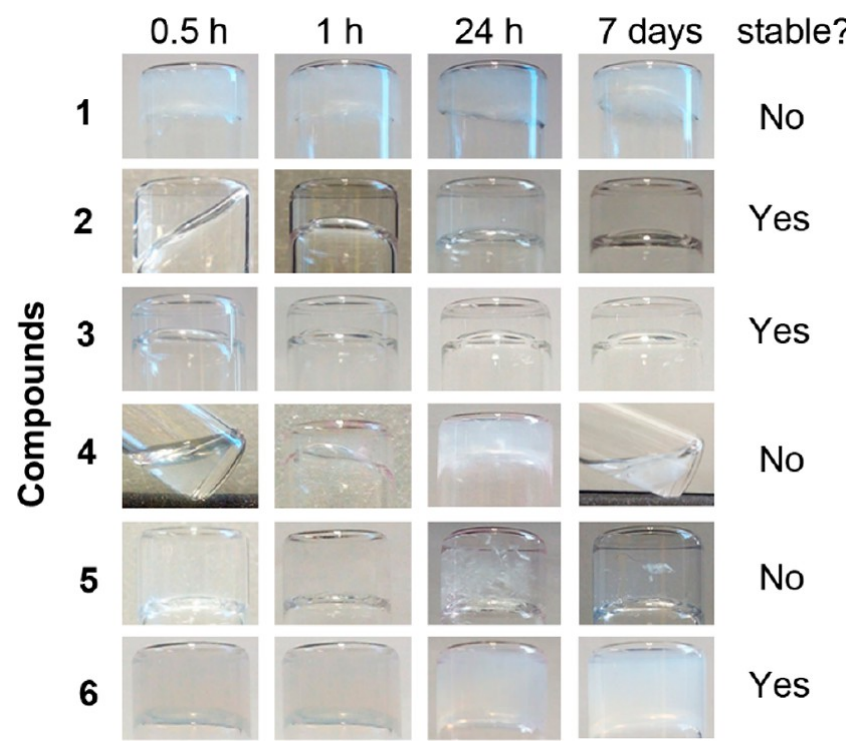

Figure 1. Photographs of supramolecular hydrogels of compounds 1-6 at their mgc and hydrogel stability over 7 days (i.e., no transition to other phases).

resistance to applied stress increased with heterochirality and halogenation (see Supporting Information, Section S8). Compound 1 displayed a gel-to-sol transition at just 0.02
$\mathrm{kPa}$, whereas its diastereomer 2 transitioned at $0.15 \mathrm{kPa}$, similarly to halogenated $4-6(0.11 \mathrm{kPa})$ despite their lower mgc. Ortho-fluorination in 3 led to an intermediate stress resistance $(0.07 \mathrm{kPa})$.

The hydrogels were tested for thermal reversibility. Compounds $\mathbf{1 - 5}$ started to disassemble at $42-47^{\circ} \mathrm{C}$, whereas iodinated 6 showed higher thermal stability until $74{ }^{\circ} \mathrm{C}$. Interestingly, homochiral 1 did not recover after melting, whereas all heterochiral dipeptides $\mathbf{2}-\mathbf{6}$ recovered within a few minutes (see Supporting Information, Section S7) with iodinated 6 showing the fastest recovery. TEM micrographs revealed anisotropic morphologies in all cases (Figure 2). Homochiral 1 formed rigid microtubes, as reported previously, with heterogeneous diameters in the range of several hundreds of nanometers. After thermoreversion, sample 1 was heterogeneous with liquid separation from the solid; TEM micrographs revealed larger microtubes, thus with reduced surface area to interact with water, and this feature could explain its inability to gel. In contrast, heterochiral 2 assembled in homogeneously sized fibrils with an average diameter of 4.3 $\pm 0.6 \mathrm{~nm}(n=100)$. An analogous size distribution was observed also for $3(4.4 \pm 0.7 \mathrm{~nm})$ and $4(3.9 \pm 0.6 \mathrm{~nm})$ after thermoreversion, suggesting a similar supramolecular packing. Compounds $\mathbf{5}$ and $\mathbf{6}$ formed thicker fibers, which was not too surprising given the opacity of their corresponding hydrogels (see Supporting Information, Section S9).

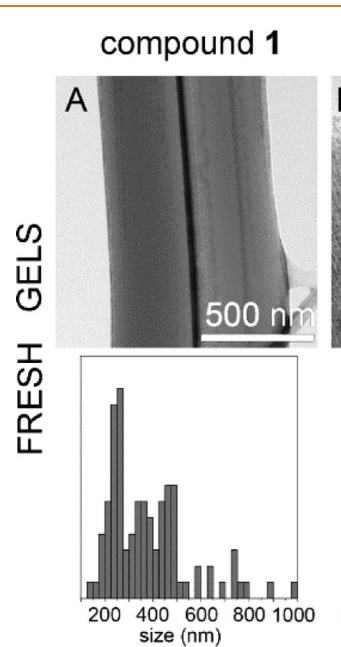

\section{compound 2}
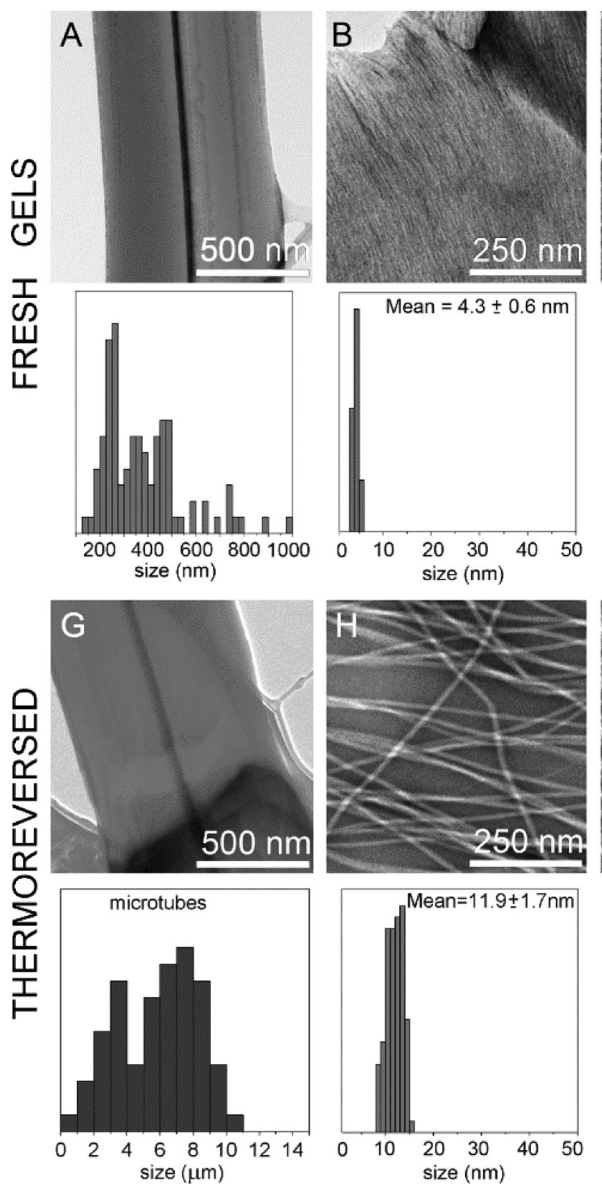
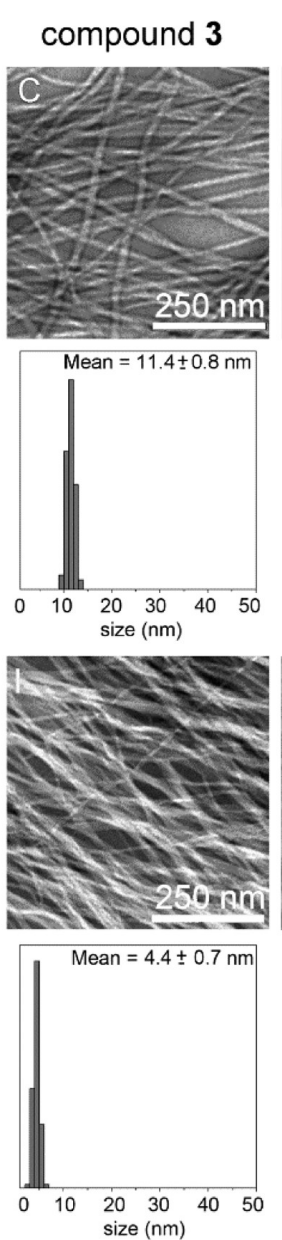

\section{compound 4}
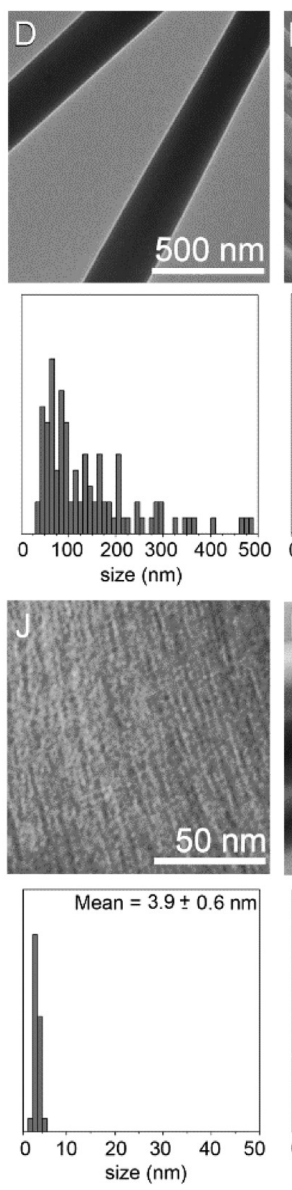

\section{compound $\mathbf{5}$}
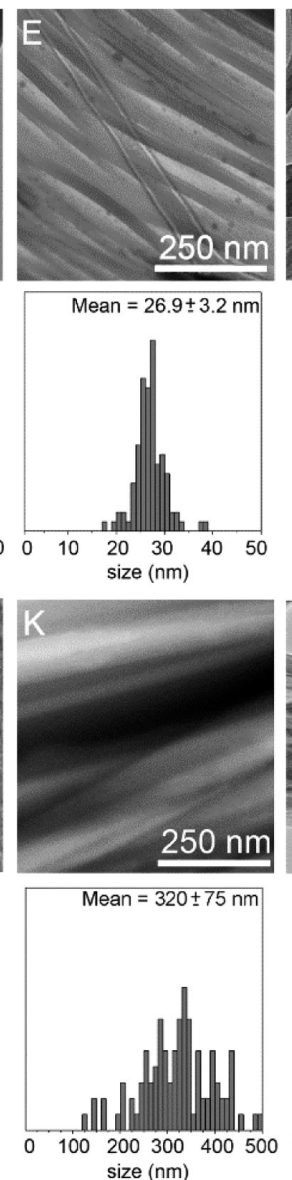

compound 6
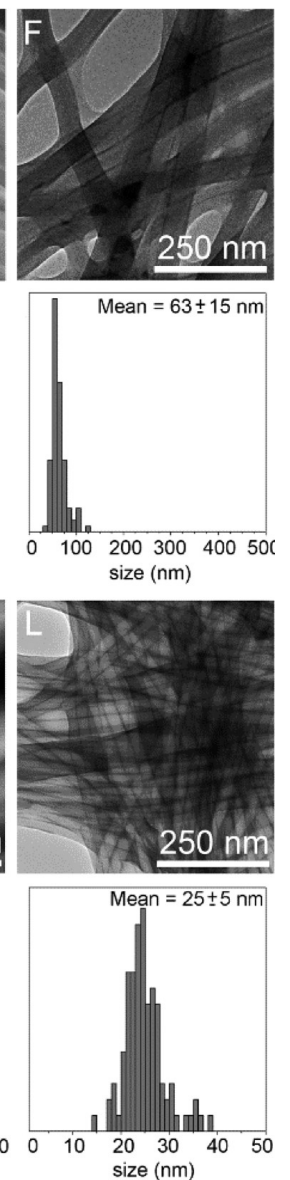

Figure 2. TEM micrographs with, underneath, the corresponding fibril diameter distribution $(n=100)$ of self-assembled $1-6$ at their mgc before $(\mathrm{A}-\mathrm{F})$ and after $(\mathrm{G}-\mathrm{L})$ thermoreversion. 
Peptide Conformations. To gain a deeper understanding of the self-assembly mechanisms of these dipeptides, their conformations were investigated spectroscopically. The circular dichroism (CD) spectrum for homochiral $\mathbf{1}$ was in agreement with the literature (Figure $3 \mathrm{~A}){ }^{28}$ Heterochiral
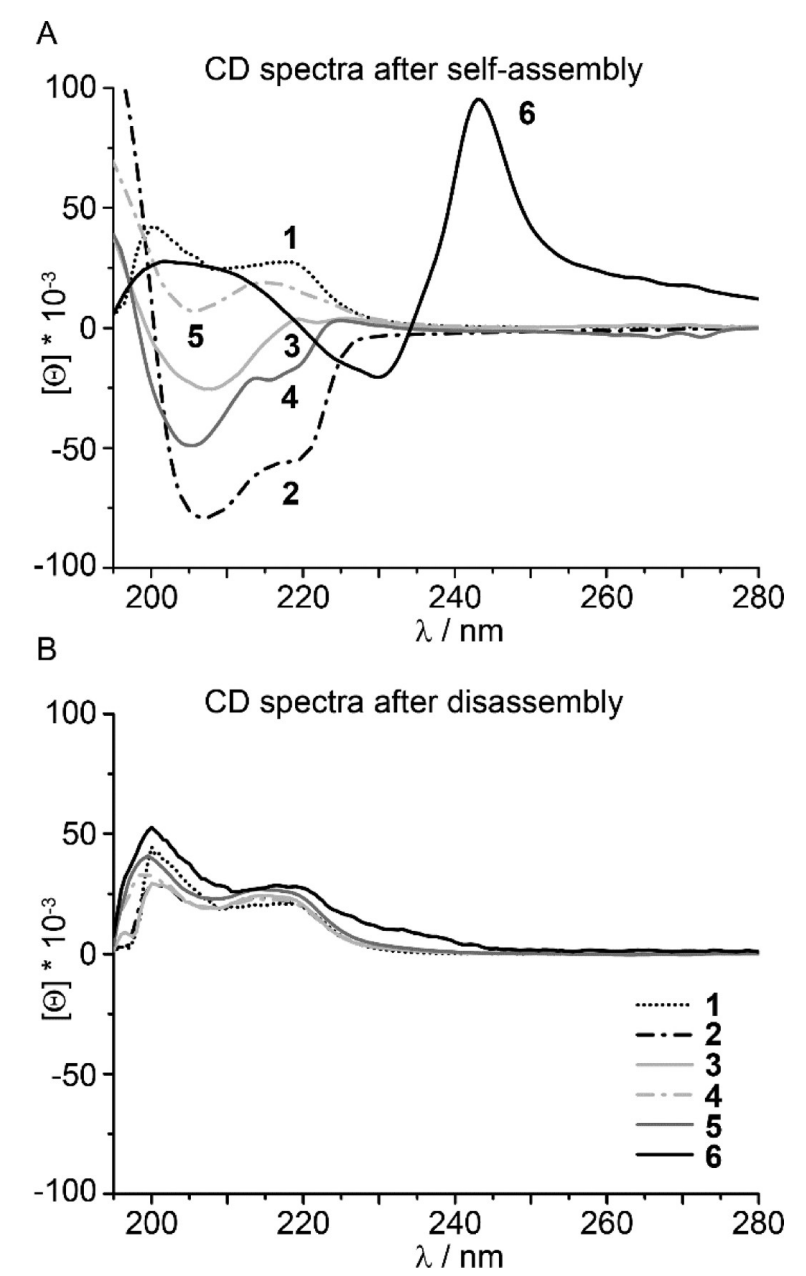

Figure 3. CD spectra for 1-6 hydrogels in the assembled state (A) and upon heating-induced disassembly at $70-80^{\circ} \mathrm{C}(\mathrm{B})$.

dipeptides 2-6 displayed diverse CD signatures in the UV region. The complex signals for the supramolecular hydrogels (Figure 3A) were the result of the self-assembly process and were challenging to interpret. However, it is worth noting that the $\mathrm{CD}$ spectrum of the gel of compound $\mathbf{6}$ was significantly different compared to all the others, being the only one displaying an unusual maximum at $243 \mathrm{~nm}$. The spectrum suggested a different packing mode relative to compounds 15. It was reminiscent of the CD signature of Asp-Phe-Asn-Lys(4-I)-Phe, an amyloidogenic gelator that did not establish halogen bonding, characterized by a cross- $\beta$-sheet structure, whereby the peptides stacked in layers with the backbones perpendicular to the long axis of the fibrils. ${ }^{26}$ Heating ramps confirmed a similar trend in melting temperatures as observed macroscopically, that is, $38-50{ }^{\circ} \mathrm{C}$ for compounds $\mathbf{1 - 5}$, and a significantly higher $T_{\mathrm{m}}$ of $60{ }^{\circ} \mathrm{C}$ for iodinated 6 (see Supporting Information, Section S10). In the disassembled state (i.e., at $70-80{ }^{\circ} \mathrm{C}$ ), all compounds $1-6$ displayed similar spectra (Figure 3B), analogous to $\mathbf{1}$ in the assembled state (Figure 3A). We inferred that all peptides adopted analogous conformations in the solution state, and the different details of their structures were amplified during the assembly process. The CD signature in the $200-220 \mathrm{~nm}$ range has been recently assigned to a distribution of conformations in solution with the most stable ones populating the top left quadrant of the Ramachandran plot (i.e., $\beta$-structures and turns). ${ }^{42}$ The amide I region of ATR-IR spectra and positive response to the Thioflavin $\mathrm{T}$ fluorescence amyloid assay showed a behavior similar to $\beta$-structures (see Supporting Information, Sections S11 and S12), although dipeptides have just one amide bond that is insufficient to satisfy the requirement of the hydrogenbonding network that defines $\beta$-sheets. ${ }^{43}$

Chirality Effects on Interactions Responsible for Hierarchical Bundling. Single-crystal XRD structures revealed that despite the different stereoconfigurations of $\mathbf{1}^{44}$ and 2 they displayed a strikingly similar supramolecular packing (Figure 4). In both cases, peptides stacked to form nanotubes, whereby the hydrogen-bond and salt-bridge patterns were conserved with similar interatomic distances. The chirality of the $\mathrm{N}$-terminal residue dictated the direction of the peptide helical arrangement along the tube, which was thus left-handed for the L-dipeptide $\mathbf{1}$ (silver in Figure 4A) and right-handed for D-L-heterochiral 2 (cyan in Figure 4A). A key difference between the two stereoisomers was the orientation of Phe aromatic side chains (Figure 4B, top) with only heterochiral 2 (cyan) displaying the Phe side chains engaged in intramolecular face-to-face interactions. The nanotube inner cavity (Figure 4B, bottom) was defined by a tubular single layer of peptide molecules arranged head-to-tail with a 6-fold helical symmetry in both $\mathbf{1}$ and $\mathbf{2}$ crystals, thus forming an amphipathic water-channel with hydrophilic interior and hydrophobic exterior.

Hydrophobic interactions involving molecules forming the water-channel further identified a tubular double layer of peptides with a total of 18 molecules visible in the top-view projection for both 1 and 2 (Figure 4C, top). However, as a result of the different spatial orientation of Phe side chains between stereoisomers $\mathbf{1}$ and 2, only in the case of homochiral 1 was the outer surface of the tubular double layer of peptides rugged with significant interdigitation between aromatic rings in steric zippers, which are an amyloid stabilizing feature ${ }^{45}$ that secured hierarchical assembly into bundles (Figure 4C, silver). By contrast, in the case of heterochiral 2, the tubular double layer of peptides displayed a smoother outer surface, thanks to a higher level of intramolecular aromatic interactions, with an outer diameter of $4.2 \mathrm{~nm}$ (cyan in Figure 4C) which matched well the average fibril size of $4.3 \mathrm{~nm}$ observed by TEM (see Figure 2B).

This difference between $\mathbf{1}$ and $\mathbf{2}$ was quantified in silico (see Supporting Information, Section S13) with the hydrophobic interface area between channels being wider for $1\left(389 \AA^{2}\right)$ than $2\left(356 \AA^{2}\right)$. A consistent trend was also seen for the binding free energy (estimated with the MM/GBSA approach) between zipping peptides of different channels for $1(-31.2$ $\mathrm{kcal} / \mathrm{mol}$ ) as compared to the non-zipping peptides of 2 $(-27.7 \mathrm{kcal} / \mathrm{mol})$. It is worth noting that the MM/GBSA approach does not fully account for entropic contributions due to solvent reorganization around the surface of the peptides. Furthermore, the change in the configurational entropy of the solute is very difficult to estimate and often it is neglected (as in this contribution). The tighter packing of homochiral 1 relative to heterochiral $\mathbf{2}$ was also consistent with a narrower 


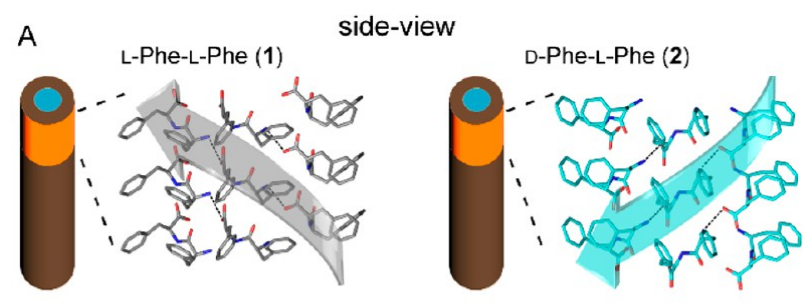

B
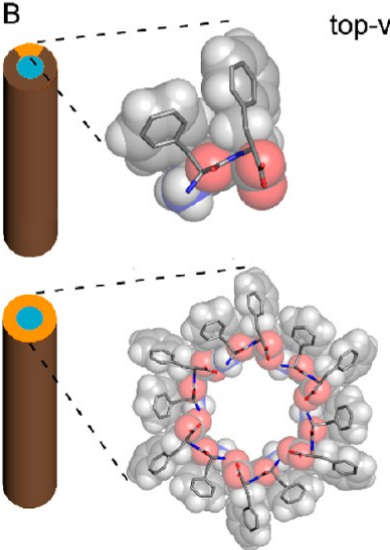

top-view
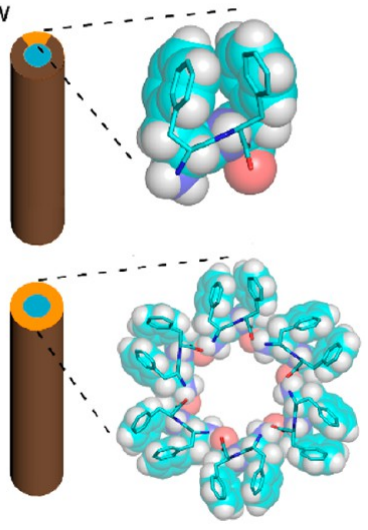

C
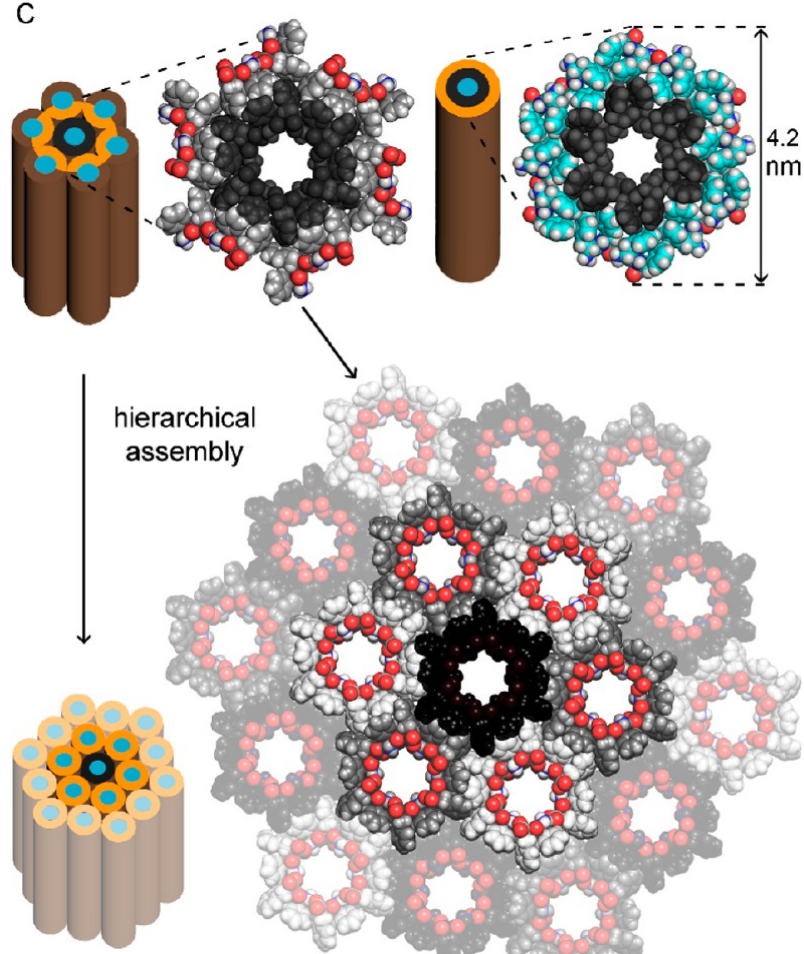

erarchica
assembly

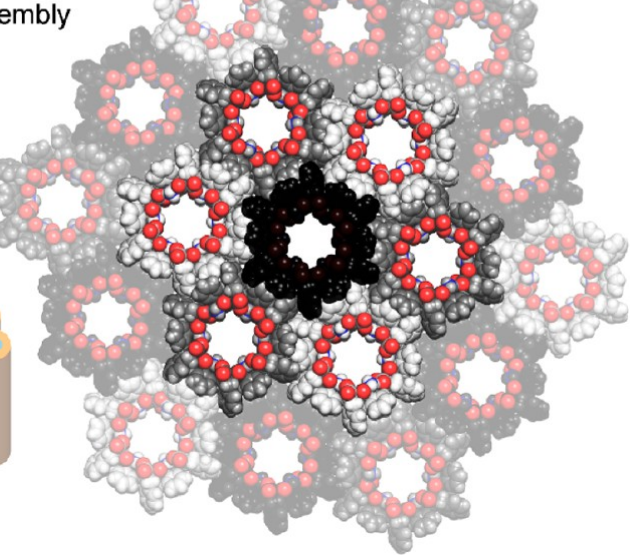

Figure 4. Single-crystal XRD structures of homochiral $1^{44}$ (silver) and heterochiral 2 (cyan, CCDC 2016375) peptide nanotubes. (A) Side-view of stacked peptides revealed opposite screw-sense for 1 (silver) and 2 (cyan), dictated by the chirality of the $\mathrm{N}$-terminal amino acids. (B) Top-view of nanotube inner cavities defined by the projection six peptide molecules arranged head-to-tail for both 1 (silver) and 2 (cyan), but only in 2 do the two Phe side chains interact face to face intramolecularly. (C) Top-view of the nanotubes identified by the projection of 18 peptide molecules for 1 (silver) showed hierarchical bundling stabilized by aromatic zippers; instead, a space-fill view of heterochiral 2 (cyan) revealed the outer diameter of a double layer of peptide molecules to agree with the diameter of fibrils measured by TEM. solvent-accessible surface area (SASA) corresponding to $74 \AA^{2}$ for 1 against $199 \AA^{2}$ for 2 .

These analyses explained well why homochiral peptide 1 assembly extended to microtubes, whereas the heterochirality of 2 oriented Phe side chains to avoid hierarchical bundling and instead stabilize double layers of peptides constituting 4 nm-wide fibrils. In light of the well-known challenges to control the growth of hierarchical assemblies of flexible simple molecules, such as unprotected dipeptides, this dataset revealed a promising yet very simple strategy to control selforganization to yield a highly homogeneous population of nanofibrils.

Fluorination Effects on Nanotube Hierarchical Assembly. Single-crystal structures of halogenated heterochiral dipeptides confirmed that the zwitterionic state was essential but not sufficient for their self-assembly into water-channels. In particular, ionic interactions between termini observed in the head-to-tail packing shown in Figure 4A,B could not be established in the crystal structure of the cationic form of fluorinated 4 (CCDC 2016372, see Supporting Information, Section S14).

In contrast, the zwitterionic states of fluorinated heterochiral dipeptides could engage in interactions and packing analogous to those described above for heterochiral 2 , as confirmed by the crystal structure of fluorinated $\mathbf{5}$ (Figure $5 \mathrm{~A}$ ), as well as powder-XRD diffraction analysis of aged gels, whose patterns matched well those generated from the single-crystal structures of the corresponding zwitterions (see Supporting Information, Section S15). Compound 5 was chosen among the fluorinated isomers as a model for in silico studies, being the only fluorinated compound whose single-crystal XRD structure was solved for the zwitterionic state, as well as the only one whose single crystals grew from the hydrogel, thus providing a good link between the gel and crystal states. Calculations of the interfacial hydrophobic area and binding energy between peptides of different water-channels $\left(368 \AA^{2}\right.$ and $-30.6 \mathrm{kcal} /$ mol, respectively), and SASA ( $\left.89 \AA^{2}\right)$, confirmed that these parameters for fluorinated 5 were intermediate between those of homochiral $\mathbf{1}$ and heterochiral $\mathbf{2}$. These calculations agreed with the observation of an intermediate level of hierarchical bundling of the water-channels into $27 \mathrm{~nm}$ wide fibrils observed by TEM (Figures 5A and 2E). Similarly, the fibrils formed by analog 3 (Figure 2C) displayed an average diameter that corresponded in size to an assembly of 19 water-channels (Figure 5B and Supporting Information, Section S9), which, however, did not persist following thermoreversion. Thereafter they formed again the $4 \mathrm{~nm}$-wide individual fibrils analogously to isomer 4 (Figure 2I,J, and Supporting Information, Section S9), corresponding to a double layer of peptides as shown in Figure 5C.

lodination Disrupted Nanotube Self-Assembly. Iodine appeared too bulky to fit in the supramolecular water-channel organization identified by XRD for compounds $\mathbf{1 - 5}$ (shown in Figures 4 and 5). Indeed, iodinated $\mathbf{6}$ displayed a different mode of packing with layers (Figure 6A) of stacks of peptides (Figure 6B). The aromatic side chains of facing dipeptides defined hydrophobic regions that were occupied by the iodine atoms, similarly to iodinated L-diphenylalanines. ${ }^{29}$ The stabilizing effect of the interdigitation of the iodinated aromatic rings (Figure 6C) was likely the reason for the superior gelling ability of iodinated 6 relative to the other compounds $1-5$. Surprisingly, no evidence for halogen-bonding was observed, as no atoms were located within van der Waals radius of iodine, 
A D-(4-F)-Phe-L-Phe (5)
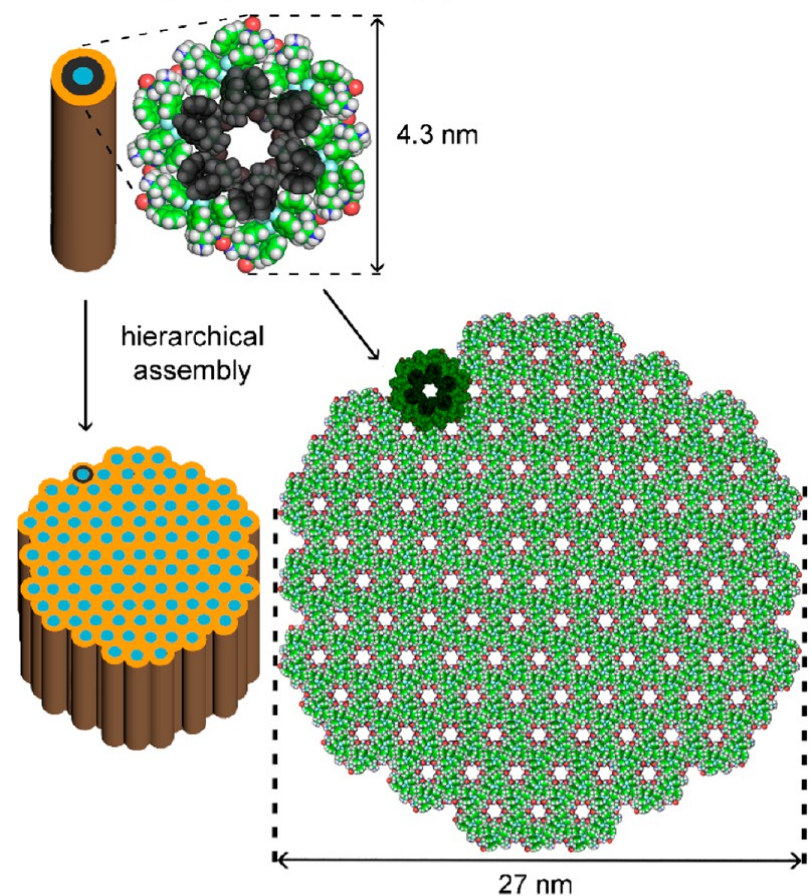

B D-(2-F)-Phe-L-Phe (3)
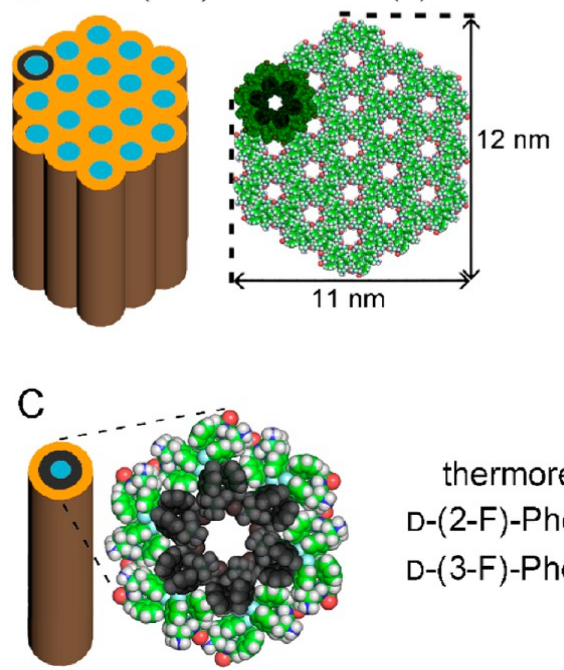

thermoreversed

D-(2-F)-Phe-L-Phe (3)

D-(3-F)-Phe-L-Phe (4)

Figure 5. (A) Single-crystal XRD structure (CCDC 2016373) of fluorinated heterochiral 5 revealed analogous packing to heterochiral 2 (compare with Figure 4C) with an intermediate level of bundling to generate $27 \mathrm{~nm}$-wide fibrils as observed by TEM. (B,C) Powder XRD on microcrystals grown on hydrogels of 3-4 confirmed packing analogous to 5 . (B) Fibrils of 3 had a diameter of 11-12 nm by TEM, corresponding to the packing of 19 water-channels. (C) Thermoreversion stabilized $4 \mathrm{~nm}$ wide fibrils for both 3 and 4, corresponding to a double-layer of peptides around a water channel as revealed by the single-crystal XRD of 5.

and no Lewis partner was oriented in the surroundings at a suitable angle. ${ }^{46}$ This is in marked contrast with L-(4-I)-Phe-LPhe and L-(4-I)-Phe-L-(4-I)-Phe crystal structure, where iodine atoms engaged in halogen bonding, yet no gel was formed. ${ }^{29}$ However, examples of amyloidogenic short peptides with (4I)-Phe not engaging in halogen bonding were also described,
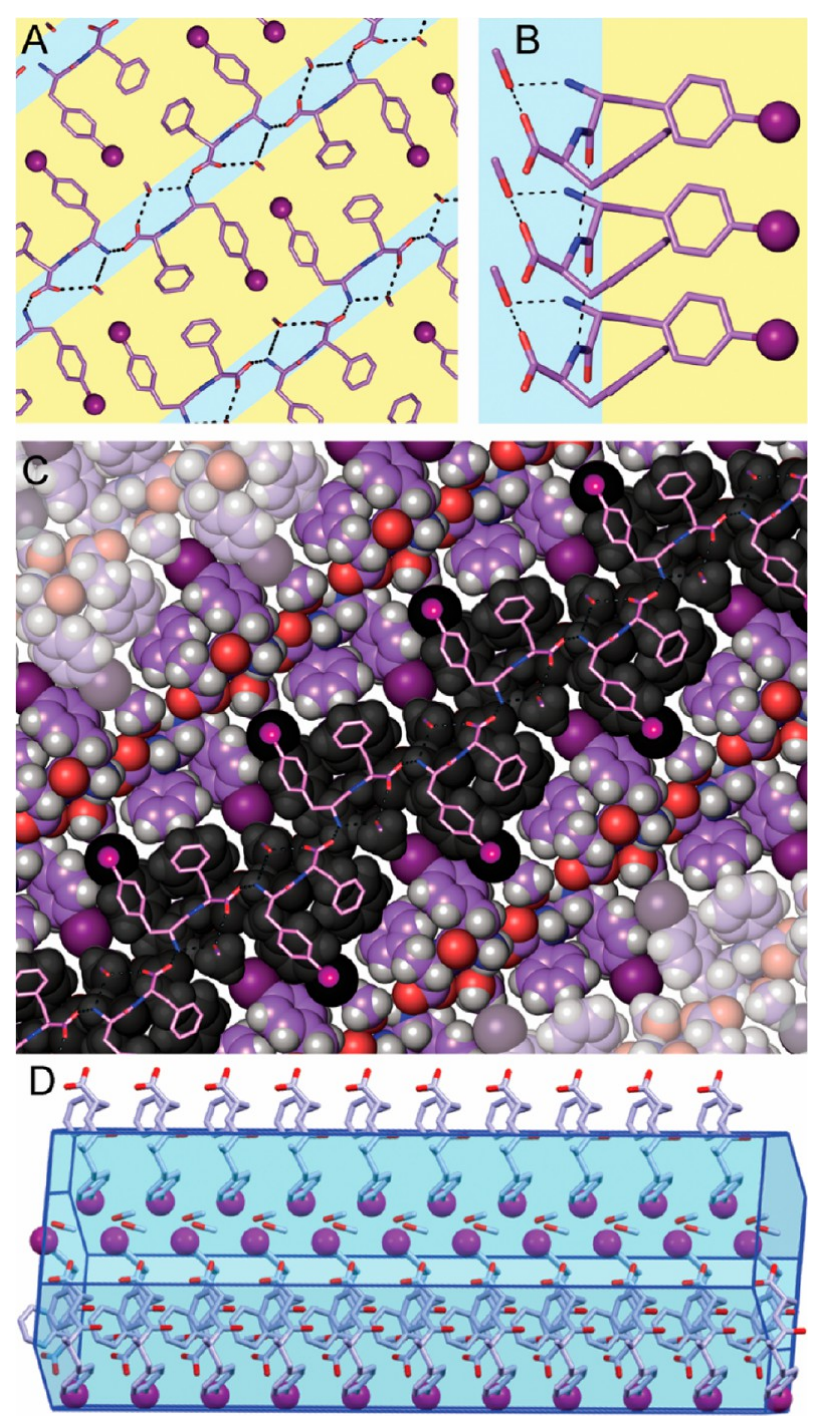

Figure 6. Single-crystal XRD structure of iodinated 6 (CCDC 2016374). (A) Hydrogen bonding and ionic interactions (dotted lines) defined hydrophilic layers (light blue), whereas iodine (purple sphere) allowed interdigitation of Phe side chains in hydrophobic layers (yellow). (B) Side-view of amphiphilic peptide stacks. Iodine atoms are located $4.9 \AA$ from each other. (C) Spacefill view of peptide layers shown in panel $A$ to show the tight supramolecular packing. (D) Side-view of peptide stacks reveals peptide backbones perpendicular to the preferential growth direction of the crystals.

whereby peptides stacked with their backbones perpendicular to the long axis of the assemblies growth, similarly to compound 6 (Figure 6D). ${ }^{26,30}$

There is an ongoing debate as to the relevance of the packing observed in the crystalline state relative to the hydrogel, as the two forms are clearly different at the macroscopic and microscopic level. Depending on the nature of the solvent and the solute, in some cases both the crystal state and the hydrogel state can act as thermodynamic systems. ${ }^{47}$ In other cases, the hydrogel is the kinetic product, and the crystal is the thermodynamic one. ${ }^{2}$ To shed light on the presence of halogen bonding in the gel state, we thus performed micro-Raman analysis (Figure 7). Raman spectra of 6 in the crystal and gel states were nearly identical, suggesting 

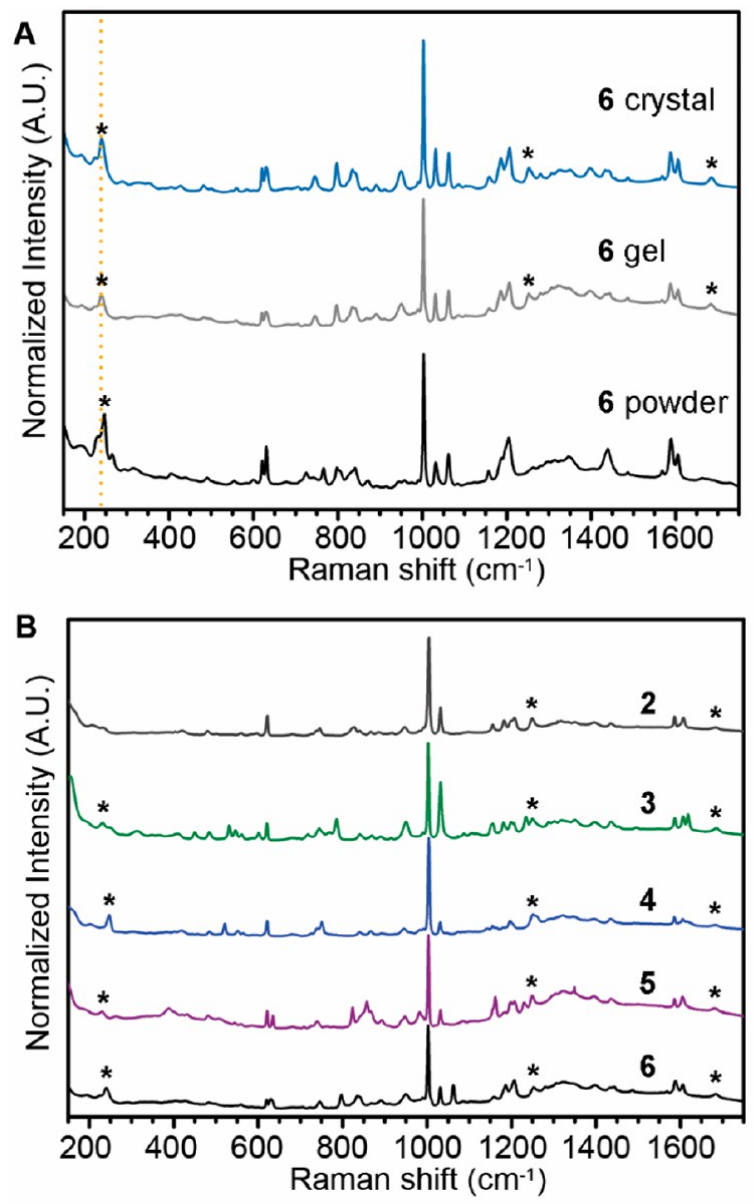

Figure 7. (A) Raman spectra of iodinated 6 in the crystal, gel, and powder forms. The dotted orange line marks the $\mathrm{C}-\mathrm{I}$ stretch. (B) Raman spectra of compounds $2-6$ in the gel form. * denotes significant differences that were inferred to arise from supramolecular organization.

analogous packing. By contrast, diagnostic differences were noted with the powder (signals marked with $*$ in Figure $7 \mathrm{~A}$ ).

The presence of iodine in 6 resulted in three additional bands relative to 2 , due to the $\mathrm{C}-\mathrm{I}$ stretching $\left(246 \mathrm{~cm}^{-1}\right)$, ring asymmetric torsion $\left(630 \mathrm{~cm}^{-1}\right)$ and ring vibration (1062 $\mathrm{cm}^{-1}$ ) of the iodinated benzene ring (Figure 7B). ${ }^{48,49}$ The former of the three bands displayed a $6 \mathrm{~cm}^{-1}$ red shift in the gel and crystal, relative to the powder form. Because no halogen bond was observed in the crystal, and because Raman spectra of the crystal and the gel state displayed analogous signature, we inferred that the red shift was simply a manifestation of the hydrophobic environment surrounding the iodine atom as a result of self-assembly. ${ }^{50,51}$ In the amide I region, the signal at $1664 \mathrm{~cm}^{-1}$ in the powder was shifted to $1685 \mathrm{~cm}^{-1}$ in the gel and crystal states, as a result of the extended hydrogen-bonding pattern between amides. ${ }^{52,53}$ Similarly, the signal at $1250 \mathrm{~cm}^{1}$ in the amide III region, resembling the $\beta$-sheet signature, was clearly visible in the gel and crystal states. ${ }^{52,53}$ Analysis of the other gels confirmed presence of the diagnostic signals due to supramolecular organization (Figure 7B).

Hydrogels in Cell Culture. The hydrogels were tested for cytotoxicity in cell culture (Figure 8). Heterochirality was confirmed to stabilize self-assembly into hydrogels. The metastable gel of L-Phe-Phe 1 did not last for $24 \mathrm{~h}$ in cell
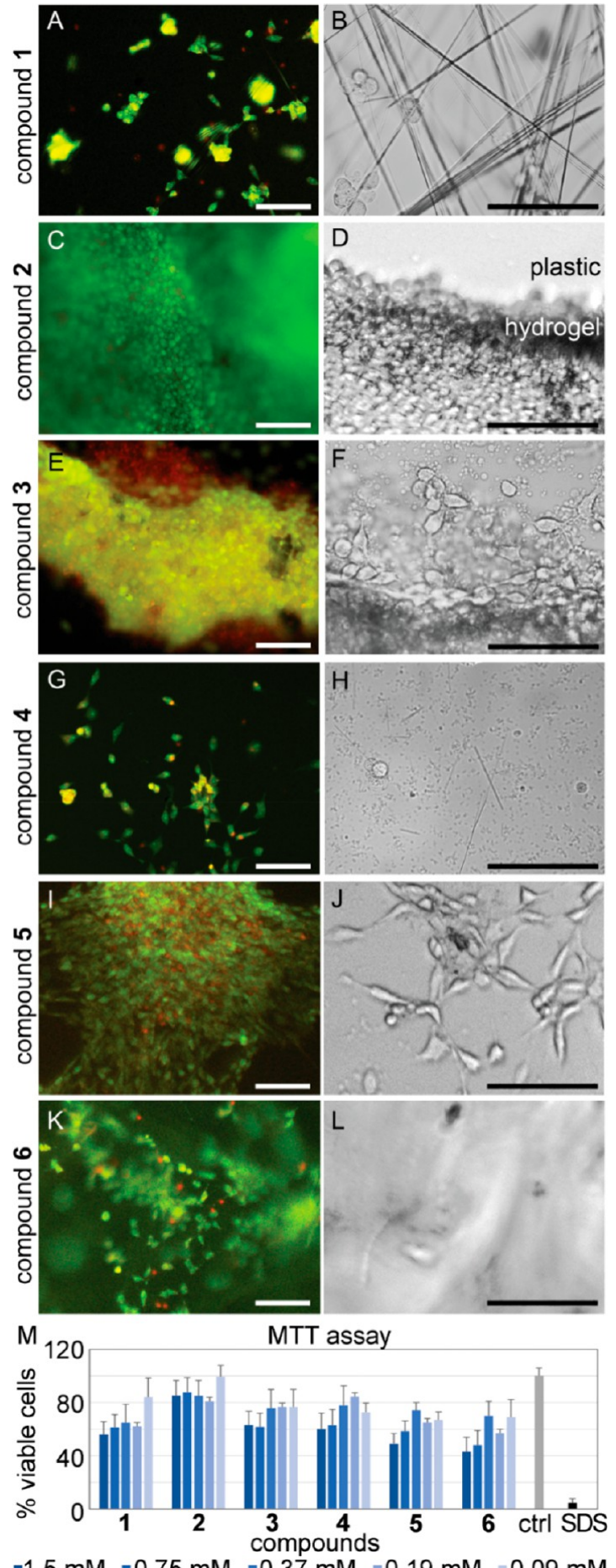

$=1.5 \mathrm{mM}=0.75 \mathrm{mM}=0.37 \mathrm{mM}=0.19 \mathrm{mM}=0.09 \mathrm{mM}$

Figure 8. (A-L) Live (green)/dead (red) fluorescence (left,) and bright-field (right) microscopy images of fibroblasts cultured on hydrogels 1-6. Scale bars $=100 \mu \mathrm{m}$. (M) Quantitative MTT assay on keratinocytes incubated with peptides in solution.

culture, and the characteristic microtubes with a few round cells were observed at the high concentration of $35 \mathrm{mM}$ (Figure 8A,B), whereas at $25 \mathrm{mM}$ microtubes were not observed by the end of the experiment. By contrast, heterochiral 2 formed a homogeneous hydrogel sheet that detached from the plastic dish, and onto which cells densely grew with high viability and integrated into the matrix as in a 
tissue, both at 25 and $35 \mathrm{mM}$ (Figure 8 and Supporting Information, Section S16).

Fibroblasts preferred to grow in the hydrogel $\mathbf{2}$ as opposed to the tissue-culture plastic surface, showing promise for the future development of this scaffold as a biomaterial (Figure $8 \mathrm{C}, \mathrm{D}$ and Supporting Information). Among the halogenated analogues, fluorinated 3-5 did not persist as hydrogels in cell culture, and cell viability was negatively affected (Figure $8 \mathrm{E}-\mathrm{J}$ ) with cell debris clearly visible in all cases (Figure $8 \mathrm{~F}, \mathrm{H}, \mathrm{J}$ ). Finally, fibroblasts grew in the hydrogel of iodinated 6, although with suboptimal viability (Figure $8 \mathrm{~K}$ ). This scaffold was the most durable as it was intact and attached to the plastic bottom after $24 \mathrm{~h}$ of cell culture, yet its translucent nature somewhat impeded bright-field visualization of cells (Figure $8 \mathrm{~L}$ ). Overall, compound 2 appeared to be the most promising biomaterial scaffold, as confirmed by quantitative MTT assay in solution on a second cell line (Figure $8 \mathrm{M}$ ). Future studies will focus on its optimization and enrichment with bioactive cues to direct cell behavior.

\section{CONCLUSIONS}

This work focused on a building block as simple as the unprotected dipeptide D-Phe-L-Phe (compound 2) and deciphered how and why heterochirality of dipeptides works as an effective design strategy to generate hydrogel biomaterials. Our studies revealed key insights that allowed control over hierarchical assembly into microstructures. Singlecrystal XRD studies revealed that despite having the opposite stereoconfiguration at one stereocenter, 2 self-organized into nanotubes with the same overall supramolecular arrangement as L-Phe-L-Phe (1), although the N-terminal amino acid stereoconfiguration dictated the screw-sense handedness form $\mathrm{N}$ - to C-terminus to be left-handed for $\mathbf{1}$ and right-handed for 2. Relative to $\mathbf{1}$, heterochiral $\mathbf{2}$ displayed increased intramolecular hydrophobic contact area between Phe side chains at the expense of the interchannel hydrophobic contact and of the consequent fibril bundling. The net result was that while LPhe-L-Phe (1) is notorious for forming heterogeneously sized microtubes, heterochiral 2 self-organized into homogeneous and transparent hydrogels composed by $4 \mathrm{~nm}$ wide fibrils, arising from a double layer of peptides around a water-filled channel. Importantly, heterochirality resulted in thermoreversibility and complete alleviation of the well-known cytotoxicity of diphenylalanine, as observed in fibroblast and keratinocyte cell culture.

Halogenation likewise proved an effective strategy to influence supramolecular organization. In particular, fluorination preserved dipeptide packing into water channels as observed for non-halogenated $\mathbf{1}$ and $\mathbf{2}$ and provided an intermediate level of intra- versus inter-molecular hydrophobic interactions, thus resulting in an intermediate level of bundling into fibrils, and hence influencing hydrogel viscoelastic properties, depending on the positioning of the fluorine atom. XRD analyses confirmed that the zwitterionic state was necessary but not sufficient for self-organization of the unprotected dipeptides into nanotubes. Interestingly, $p$ iodination of D-Phe-L-Phe completely altered the packing from water channels to amphipathic layers, yet it promoted self-assembly and zipping and yielded overall higher stability for hydrogel 6, in agreement with the literature on iodinated peptides. ${ }^{26,29}$ We note, however, that the previously reported iodinated homochiral dipeptide analogs displayed limited solubility as a result of iodination and were not reported to form gels. ${ }^{29}$ Counterintuitively, single-crystal XRD and microRaman analyses revealed no halogen bonding occurring in the present case, suggesting a mere increase in hydrophobicity and steric hindrance that stabilized the interdigitation of Phe zippers at the basis of hydrogel stability for 6 .

When tested in cell culture, fluorinated compounds 3-5 were unstable and displayed limited cytocompatibility as evidenced by reduced cell numbers, presence of dead cells, and evident cell debris. Iodinated $\mathbf{6}$ was the most stable hydrogel also in cell culture conditions, yet its translucent nature and limited cytocompatibility indicated it is not promising as a biomaterial scaffold. In contrast, hydrogels of D-Phe-L-Phe (2) formed a continuous sheet that detached from the bottom of the wells and was of preference for cells to proliferate in high numbers over the plastic found underneath. Future studies will focus on the optimization of scaffold 2 toward the inclusion of bioactive motifs to direct cell fates, thus preparing advanced, yet low-cost, biomaterials.

\section{MATERIALS AND METHODS}

All solvents were purchased of peptide-synthesis grade from Merck (Italy). O-Benzotriazole- $N, N, N, N^{\prime}$-tetramethyl-uronium-hexafluoro phosphate (HBTU) as coupling agent, 2-chlorotrityl chloride resin, Fmoc-phenylalanine-loaded Wang resin, and D-phenylalanine were obtained from GL Biochem (Shanghai, China). 1-Hydroxy-7azabenzotriazole (HOAt) was purchased from ChemPep (Wellington, FL. U.S.A.). All of the other chemicals were obtained from Merck (Italy). Milli-Q water (MQ water) was collected from a Millipore RiOs/Origin apparatus with a resistivity greater than $18 \mathrm{M} \Omega \mathrm{cm}$. Peptides were synthesized on solid-phase by using standard procotols for Fmoc-protected amino acids (Sigma-Aldrich, Italy). The crude peptides were purified by reverse-phase HPLC using a semipreparative C18-column (Kinetex, $5 \mu \mathrm{m}, 100 \AA \AA, 250 \times 10 \mathrm{~mm}$, Phenomenex) on an Agilent 1260 Infinity apparatus with a photodiode array detector (G1315C), and autosampler (G1329B). Acetonitrile $(\mathrm{MeCN}) /$ water with $0.1 \%$ TFA was used as an eluent mixture with the following program for the compounds 1-5: $t=0-2$ min. $30 \% \mathrm{MeCN} ; t=12$ min. $55 \% \mathrm{MeCN} ; t=15 \min .95 \% \mathrm{MeCN}$. Instead, the following was used for compound 6: $t=0-2 \mathrm{~min} .30 \%$ $\mathrm{MeCN} ; t=12$ min. $42 \% \mathrm{MeCN} ; t=15$ min. 95\% MeCN. The product fractions were lyophilized and analyzed for identity and purity by LC-MS using an Agilent 6120 single quadrupole system. ${ }^{1} \mathrm{H}$ - and ${ }^{13} \mathrm{C}$ NMR spectra were registered on a Varian Innova Instrument with chemical shift reported as ppm (in DMSO or MeCN with tetramethylsilane as internal standard).

Hydrogel Formation. Dipeptides were dissolved at various concentrations $(2 \times$ the final concentration indicated in the manuscript for the various tests) in alkaline sodium phosphate buffer (0.1 $\mathrm{M}, \mathrm{pH} 11.8$ ) with the aid of sonication in a Branson ultrasound bath $40 \mathrm{kHz}$ at room temperature. Hydrogelation was then triggered by lowering the $\mathrm{pH}$ of the solution to physiological neutral $(7.3 \pm 0.1)$ with the addition of an equal volume of a mildly acidic sodium phosphate buffer (0.1 M, pH 5.8). All buffer solutions were filtered $(0.2 \mu \mathrm{m})$ prior to use.

Oscillatory Rheology. All hydrogels were analyzed freshly made on a Kinexus Plus (Malvern) with a stainless steel parallel-plate geometry at $25{ }^{\circ} \mathrm{C}$ (Peltier) with a gap of $1 \mathrm{~mm}$. Time sweeps were collected at $1 \mathrm{~Hz}$ and $1 \mathrm{~Pa}$, frequency sweeps at $1 \mathrm{~Pa}$ and stress sweeps at $1 \mathrm{~Hz}$. Different replicas were run for each experiment, and representative data are shown. Rheological data and a table summary with average \pm standard deviation for $G^{\prime}$ and $G^{\prime \prime}$ can be found in the Supporting Information Section S8.

Circular Dichroism. Hydrogels were formed in situ as described above in a $0.1 \mathrm{~mm}$ thick quartz cuvette on a Jasco J-815 spectropolarimeter with $1 \mathrm{~s}$ integrations, a step size of $1 \mathrm{~nm}$, and a bandwidth of $1 \mathrm{~nm}$ at $25^{\circ} \mathrm{C}$ (Peltier). Heating ramps were performed with $5{ }^{\circ} \mathrm{C}$ intervals and a heating ramp of $5{ }^{\circ} \mathrm{C} \mathrm{min}^{-1}$. 
ATR FT-IR. Hydrogels were formed in a glass vial, and a small portion was transferred onto a $1 \mathrm{~cm}^{2}$ silicon wafer and left to dry in vacuo for $24 \mathrm{~h}$. Spectra were acquired at resolution of $4 \mathrm{~cm}^{-1}$ and 128 scans on a PerkinElmer System 2000 with a Ge crystal.

Thioflavin T Fluorescence. The assay was performed in an Infinite M1000 pro (Tecan) with Greiner 96 U Bottom Black Polystyrene plates (VWR) on $150 \mu \mathrm{L}$ hydrogels prepared in the wells $(n=6)$. After $1 \mathrm{~h}$ of assembly, $30 \mu \mathrm{L}$ of a solution of Thioflavin $\mathrm{T}$ $(29.1 \mu \mathrm{M}$ in $20 \mathrm{mM}$ glycine- $\mathrm{NaOH} \mathrm{pH} 7.5$, filtered with a $0.2 \mu \mathrm{m}$ filter) was added to the wells. Fluorescence emission was analyzed after 15 min using $446 \mathrm{~nm}$ as the excitation wavelength and $490 \mathrm{~nm}$ as the emission wavelength (with a $20 \mathrm{~nm}$ bandwidth).

TEM Analysis. TEM analyses were acquired on Jeol JEM 2100 (Japan) at $100 \mathrm{kV}$. TEM grids (SPI) were exposed to UV-Ozone Procleaner Plus for $30 \mathrm{~min}$. After $24 \mathrm{~h}$ of self-assembly, hydrogels were deposited onto the grids, then they were dried for $15 \mathrm{~min}$ at room temperature and contrasted by aqueous tungsten phosphate solution (filtered: $0.2 \mu \mathrm{m}$; $\mathrm{pH} 7.4$ ).

Crystallization. Crystals of the different compounds were grown with the vapor diffusion method as described previously. ${ }^{42}$ Compounds were dissolved in methanol (compound 2 at $2.5 \mathrm{mM}$; compounds 4-6 at $2 \mathrm{mM}$ ). Eight hundred microliters of solution was deposited and put in vapor diffusion with a reservoir containing $3 \mathrm{~mL}$ of a mixture of methanol and water (10/90 for compounds $\mathbf{2}$ and $\mathbf{6}$, and 50/50 for compounds 4 and 5). Single crystals were analyzed at Elettra Synchrotron XRD1 beamline. See Supporting Information, Section S14, for further details.

Raman Spectroscopy. Raman spectra were acquired using a 785 $\mathrm{nm}$ laser in an Invia Renishaw microspectrometer (50). For each sample, at least 10 spectra $\left(1 \mathrm{~cm}^{-1}\right.$ resolution) were collected to verify homogeneity. In the case of the hydrogels, samples were transferred to a silicon wafer and dried under vacuum before the analysis to yield xerogels.

Fibroblast Cell Culture. All solutions were sterilized with $0.2 \mu \mathrm{m}$ filter prior to use and gels were prepared by following the procedure indicated above. Each gel $(20 \mu \mathrm{L} /$ well $)$ was carefully formed in the microwells of a $\mu$-Slide for Angiogenesis uncoated slide (Ibidi, Germany) according to the manufacturer's instructions and left at room temperature for $24 \mathrm{~h}$. Thirty microliters of culture media (DMEM $+10 \%$ fetal serum albumin, and $2 \%$ antimycotic and antibiotic from GIBCO) was added on the top of each gel for $1 \mathrm{~h}$. The solution was then carefully removed and NIH3T3 fibroblasts were added to the gels ( 10000 cells per well, $30 \mu \mathrm{L}$ of media), and cultured at $37^{\circ} \mathrm{C}, 5 \% \mathrm{CO}_{2}$ for $24 \mathrm{~h}$, by handling the slides according to the manufacturers' instructions. Cell viability was analyzed by using acridine orange ( $5 \mu \mathrm{L} /$ well of a $20 \mu \mathrm{M}$ solution in $50 \mathrm{mM}$ PBS) and propidium iodide ( $4 \mu \mathrm{L} /$ well of a $30 \mu \mathrm{M}$ solution in $50 \mathrm{mM}$ PBS) as dyes. After $15 \mathrm{~min}$ of incubation at $37{ }^{\circ} \mathrm{C}$, cells were imaged with a Leica microscope (DFC450C; software LASV4.13) with bright-field and fluorescence green filter (ex. 450-490 nm, em. > 520 nm) with $10 \times$ and $40 \times$ objectives. Each condition was repeated at least in triplicate, and the experiment was repeated twice.

MTT Assay. HaCaT cells (keratinocytes) were seeded (10000 cells per well) on 96-well microplates (tissue-culture grade, clear, flat bottom) in $100 \mu \mathrm{L}$ of medium (DMEM+ $10 \% \mathrm{FCS}$, and $2 \%$ antimycotic and antibiotic) and incubated overnight at $37{ }^{\circ} \mathrm{C}$ and $5 \%$ $\mathrm{CO}_{2}$. The medium was removed and exchanged with $100 \mu \mathrm{L}$ of medium with $2 \%$ DMSO and serial dilutions of each peptide concentration. One percent SDS served as positive control (death). Cells were cultured for $24 \mathrm{~h}$, then $10 \mu \mathrm{L}$ of the MTT labeling reagent (Sigma, $0.5 \mathrm{mg} \mathrm{mL}^{-1}$ ) was added, and the microplate was incubated for $4 \mathrm{~h}$ in a humidified atmosphere $\left(37^{\circ} \mathrm{C}, 5 \% \mathrm{CO}_{2}\right)$. Next, $100 \mu \mathrm{L}$ of the solubilization solution for formazan crystals $(4 \mathrm{mM} \mathrm{HCl}+0.1 \%$ IGEPAL in isopropanol) was added to each well, and the microplate was kept at RT under shaking (Rocker-Shaker MR-12 Biosan, Vetrotecnica) for $30 \mathrm{~min}$. After visual inspection for complete solubilization of the purple formazan crystals, the absorbance was read at $570 \mathrm{~nm}$ with a reference wavelength of $690 \mathrm{~nm}$ (light scattering) using a microplate (ELISA) reader (Synergy H1 Hybrid reader-
BioTeK). Three independent experiments were run in duplicate $(n=$ 6).

Molecular Models. Molecular models of 1, 2, and 5 were generated starting from the X-ray structures. Namely, periodic systems containing three unit cells running along the $z$-direction were generated using CHIMERA. ${ }^{54}$ Systems were solvated with water using the AMBER18 (D. A. Case et al. AMBER 2018 (University of California, San Francisco)) tools and the structures were optimized using restraints on non-hydrogenous atoms. Solvation binding free energies were calculated on optimized structures using the MM/ GBSA approach, ${ }^{55}$ whereas the interface areas were estimated using the Intersurf tool of CHIMERA. See Supporting Information, Section S13, for details.

\section{ASSOCIATED CONTENT}

\section{sI Supporting Information}

The Supporting Information is available free of charge at https://pubs.acs.org/doi/10.1021/acsnano.0c06041.

Spectroscopic data for compounds 1-6, HPLC traces, rheology, TEM, ATR-IR, Thioflavin T fluorescence, in silico modeling and XRD data, and cell culture images (PDF)

\section{AUTHOR INFORMATION}

\section{Corresponding Authors}

Rita De Zorzi - Chemical and Pharmaceutical Sciences Department, University of Trieste, 34127 Trieste, Italy; Email: rdezorzi@units.it

Silvia Marchesan - Chemical and Pharmaceutical Sciences Department, University of Trieste, 34127 Trieste, Italy; • orcid.org/0000-0001-6089-3873; Email: smarchesan@ units.it

\section{Authors}

Slavko Kralj - Chemical and Pharmaceutical Sciences Department, University of Trieste, 34127 Trieste, Italy; Materials Synthesis Department, Jožef Stefan Institute, 1000 Ljubljana, Slovenia; ○ orcid.org/0000-0002-0771-3818

Ottavia Bellotto - Chemical and Pharmaceutical Sciences Department, University of Trieste, 34127 Trieste, Italy

Evelina Parisi - Chemical and Pharmaceutical Sciences Department, University of Trieste, 34127 Trieste, Italy

Ana M. Garcia - Chemical and Pharmaceutical Sciences Department, University of Trieste, 34127 Trieste, Italy

Daniel Iglesias - Chemical and Pharmaceutical Sciences Department, University of Trieste, 34127 Trieste, Italy; D orcid.org/0000-0002-1998-0518

Sabrina Semeraro - Chemical and Pharmaceutical Sciences Department, University of Trieste, 34127 Trieste, Italy; - orcid.org/0000-0002-3707-9117

Caterina Deganutti - Chemical and Pharmaceutical Sciences Department, University of Trieste, 34127 Trieste, Italy

Paola D'Andrea - Life Sciences Department, University of Trieste, 34127 Trieste, Italy

Attilio V. Vargiu - Physics Department, University of Cagliari, 09042 Monserrato, Italy; $\odot$ orcid.org/0000-0003-40138867

Silvano Geremia - Chemical and Pharmaceutical Sciences Department, University of Trieste, 34127 Trieste, Italy; (1) orcid.org/0000-0002-0711-5113

Complete contact information is available at: https://pubs.acs.org/10.1021/acsnano.0c06041 


\section{Author Contributions}

The manuscript was written through contributions of all authors.

\section{Notes}

The authors declare no competing financial interest.

\section{ACKNOWLEDGMENTS}

We acknowledge Elettra Sincrotrone Trieste for providing access to its synchrotron radiation facilities and we thank $M$. Polentarutti, N. Demitri, and G. Bais for assistance in using beamline XRD1. The authors acknowledge funding from the Italian Ministry of Education and Research (MIUR) through the SIR program (personal grant RBSI14A7PL to S.M.). S.K. is grateful to the European Social Fund, Operational Programme 2014-2020 (Axis 3 - Education and Training, Specific Programme no. 26 - TALENTS3 Fellowship Programme "MAGIC SPY"). We acknowledge the financial support from the Slovenian Research Agency (ARRS) for research core funding No. P2-0089, Project No. J1-7302, and the CENN Nanocenter (Slovenia) for TEM access.

\section{REFERENCES}

(1) De Santis, E.; Ryadnov, M. G. Peptide Self-Assembly for Nanomaterials: The Old New Kid on the Block. Chem. Soc. Rev. 2015, 44, 8288-8300.

(2) Wang, J.; Liu, K.; Xing, R.; Yan, X. Peptide Self-Assembly: Thermodynamics and Kinetics. Chem. Soc. Rev. 2016, 45, 5589-5604.

(3) Adams, D. J. Dipeptide and Tripeptide Conjugates as LowMolecular-Weight Hydrogelators. Macromol. Biosci. 2011, 11, 160173.

(4) Tao, K.; Levin, A.; Adler-Abramovich, L.; Gazit, E. FmocModified Amino Acids and Short Peptides: Simple Bio-Inspired Building Blocks for the Fabrication of Functional Materials. Chem. Soc. Rev. 2016, 45, 3935-3953.

(5) Du, X.; Zhou, J.; Shi, J.; Xu, B. Supramolecular Hydrogelators and Hydrogels: From Soft Matter to Molecular Biomaterials. Chem. Rev. 2015, 115, 13165-13307.

(6) Abraham, B. L.; Liyanage, W.; Nilsson, B. L. Strategy to Identify Improved N-Terminal Modifications for Supramolecular Phenylalanine-Derived Hydrogelators. Langmuir 2019, 35, 14939-14948.

(7) Wojciechowski, J. P.; Martin, A. D.; Mason, A. F.; Fife, C. M.; Sagnella, S. M.; Kavallaris, M.; Thordarson, P. Choice of Capping Group in Tripeptide Hydrogels Influences Viability in the ThreeDimensional Cell Culture of Tumor Spheroids. ChemPlusChem 2017, $82,383-389$.

(8) Truong, W. T.; Su, Y.; Gloria, D.; Braet, F.; Thordarson, P. Dissolution and Degradation of Fmoc-Diphenylalanine Self-Assembled Gels Results in Necrosis at High Concentrations in Vitro. Biomater. Sci. 2015, 3, 298-307.

(9) Martin, A. D.; Robinson, A. B.; Thordarson, P. Biocompatible Small Peptide Super-Hydrogelators Bearing Carbazole Functionalities. J. Mater. Chem. B 2015, 3, 2277-2280.

(10) Frederix, P. W.; Scott, G. G.; Abul-Haija, Y. M.; Kalafatovic, D.; Pappas, C. G.; Javid, N.; Hunt, N. T.; Ulijn, R. V.; Tuttle, T. Exploring the Sequence Space for (Tri-)Peptide Self-Assembly to Design and Discover New Hydrogels. Nat. Chem. 2015, 7, 30-37.

(11) Chan, K. H.; Xue, B.; Robinson, R. C.; Hauser, C. A. E. Systematic Moiety Variations of Ultrashort Peptides Produce Profound Effects on Self-Assembly, Nanostructure Formation, Hydrogelation, and Phase Transition. Sci. Rep. 2017, 7, 12897.

(12) Adler-Abramovich, L.; Vaks, L.; Carny, O.; Trudler, D.; Magno, A.; Caflisch, A.; Frenkel, D.; Gazit, E. Phenylalanine Assembly into Toxic Fibrils Suggests Amyloid Etiology in Phenylketonuria. Nat. Chem. Biol. 2012, 8, 701-706.

(13) Wang, Y.; Qi, W.; Huang, R.; Yang, X.; Wang, M.; Su, R.; He, Z. Rational Design of Chiral Nanostructures from Self-Assembly of a
Ferrocene-Modified Dipeptide. J. Am. Chem. Soc. 2015, 137, 78697880.

(14) Xiong, Q.; Jiang, Y.; Cai, X.; Yang, F.; Li, Z.; Han, W. Conformation Dependence of Diphenylalanine Self-Assembly Structures and Dynamics: Insights from Hybrid-Resolution Simulations. ACS Nano 2019, 13, 4455-4468.

(15) Cheng, N.; Chen, Y.; Yu, J.; Li, J. J.; Liu, Y. Photocontrolled Coumarin-Diphenylalanine/Cyclodextrin Cross-Linking of 1D Nanofibers to 2D Thin Films. ACS Appl. Mater. Interfaces 2018, 10, 68106814.

(16) Sun, H. L.; Chen, Y.; Han, X.; Liu, Y. Tunable Supramolecular Assembly and Photoswitchable Conversion of Cyclodextrin/Diphenylalanine-Based 1D and 2D Nanostructures. Angew. Chem., Int. Ed. 2017, 56, 7062-7065.

(17) Chen, J.; Qin, S.; Wu, X.; Chu, A. P. Morphology and Pattern Control of Diphenylalanine Self-Assembly via Evaporative Dewetting. ACS Nano 2016, 10, 832-838.

(18) Tao, K.; Makam, P.; Aizen, R.; Gazit, E. Self-Assembling Peptide Semiconductors. Science 2017, 358, No. eaam9756.

(19) Gan, Z.; Wu, X.; Zhu, X.; Shen, J. Light-Induced Ferroelectricity in Bioinspired Self-Assembled Diphenylalanine Nanotubes/Microtubes. Angew. Chem., Int. Ed. 2013, 52, 2055-2059.

(20) Nguyen, V.; Zhu, R.; Jenkins, K.; Yang, R. Self-Assembly of Diphenylalanine Peptide with Controlled Polarization for Power Generation. Nat. Commun. 2016, 7, 13566.

(21) Lee, J. H.; Heo, K.; Schulz-Schonhagen, K.; Lee, J. H.; Desai, M. S.; Jin, H. E.; Lee, S. W. Diphenylalanine Peptide Nanotube Energy Harvesters. ACS Nano 2018, 12, 8138-8144.

(22) Safaryan, S.; Slabov, V.; Kopyl, S.; Romanyuk, K.; Bdikin, I.; Vasilev, S.; Zelenovskiy, P.; Shur, V. Y.; Uslamin, E. A.; Pidko, E. A.; Vinogradov, A. V.; Kholkin, A. L. Diphenylalanine-Based Microribbons for Piezoelectric Applications via Inkjet Printing. ACS Appl. Mater. Interfaces 2018, 10, 10543-10551.

(23) Reches, M.; Gazit, E. Casting Metal Nanowires within Discrete Self-Assembled Peptide Nanotubes. Science 2003, 300, 625-627.

(24) Ryan, D. M.; Anderson, S. B.; Senguen, F. T.; Youngman, R. E.; Nilsson, B. L. Self-Assembly and Hydrogelation Promoted by $\mathrm{F}_{5}$ Phenylalanine. Soft Matter 2010, 6, 475-479.

(25) Ryan, D. M.; Anderson, S. B.; Nilsson, B. L. The Influence of Side-Chain Halogenation on the Self-Assembly and Hydrogelation of Fmoc-Phenylalanine Derivatives. Soft Matter 2010, 6, 3220-3231.

(26) Bertolani, A.; Pirrie, L.; Stefan, L.; Houbenov, N.; Haataja, J. S.; Catalano, L.; Terraneo, G.; Giancane, G.; Valli, L.; Milani, R.; Ikkala, O.; Resnati, G.; Metrangolo, P. Supramolecular Amplification of Amyloid Self-Assembly by Iodination. Nat. Commun. 2015, 6, 7574.

(27) Conte, M. P.; Singh, N.; Sasselli, I. R.; Escuder, B.; Ulijn, R. V. Metastable Hydrogels from Aromatic Dipeptides. Chem. Commun. 2016, 52, 13889-13892.

(28) Kurbasic, M.; Semeraro, S.; Garcia, A. M.; Kralj, S.; Parisi, E.; Deganutti, C.; De Zorzi, R.; Marchesan, S. Microwave-Assisted Cyclization of Unprotected Dipeptides in Water to 2, 5Piperazinediones and Self-Assembly Study of Products and Reagents. Synthesis 2019, 51, 2829-2838.

(29) Pizzi, A.; Catalano, L.; Demitri, N.; Dichiarante, V.; Terraneo, G.; Metrangolo, P. Halogen Bonding as a Key Interaction in the SelfAssembly of Iodinated Diphenylalanine Peptides. Pept. Sci. 2020, 112, No. e24127.

(30) Pizzi, A.; Dichiarante, V.; Terraneo, G.; Metrangolo, P. Crystallographic Insights into the Self-Assembly of KLVFF AmyloidBeta Peptides. Pept. Sci. 2018, 110, No. e23088.

(31) Vargiu, A. V.; Iglesias, D.; Styan, K. E.; Waddington, L. J.; Easton, C. D.; Marchesan, S. Design of a Hydrophobic Tripeptide that Self-assembles into Amphiphilic Superstructures Forming a Hydrogel Biomaterial. Chem. Commun. 2016, 52, 5912-5915.

(32) Garcia, A. M.; Kurbasic, M.; Kralj, S.; Melchionna, M.; Marchesan, S. A Biocatalytic and Thermoreversible Hydrogel from a Histidine-containing Tripeptide. Chem. Commun. 2017, 53, 81108113. 
(33) Melchionna, M. E.; Styan, K.; Marchesan, S. The Unexpected Advantages of Using D-Amino Acids for Peptide Self- Assembly into Nanostructured Hydrogels for Medicine. Curr. Top. Med. Chem. 2016, 16, 2009-2018.

(34) Clover, T. M.; O’Neill, C. L.; Appavu, R.; Lokhande, G.; Gaharwar, A. K.; Posey, A. E.; White, M. A.; Rudra, J. S. Self-Assembly of Block Heterochiral Peptides into Helical Tapes. J. Am. Chem. Soc. 2020 DOI: 10.1021/jacs.9b09755.

(35) Li, M.; Liu, M.; Shang, Y.; Ren, C.; Liu, J.; Jin, H.; Wang, Z. The Substitution of a Single Amino Acid with Its Enantiomer for Control over the Adjuvant Activity of Self-Assembling Peptides. RSC Adv. 2020, 10, 13900-13906.

(36) Insua, I.; Montenegro, J. 1D to 2D Self Assembly of Cyclic Peptides. J. Am. Chem. Soc. 2020, 142, 300-307.

(37) Bolt, H.; Williams, C.; Brooks, R.; Zuckermann, R.; Cobb, S.; Bromley, E. Log D versus HPLC Derived Hydrophobicity: The Development of Predictive Tools to Aid in the Rational Design of Bioactive Peptoids. Biopolymers 2017, 108, No. e23014.

(38) Das, B. K.; Pramanik, B.; Chowdhuri, S.; Scherman, O. A.; Das, D. Light-Triggered Syneresis of a Water Insoluble Peptide-Hydrogel Effectively Removes Small Molecule Waste Contaminants. Chem. Commun. 2020, 56, 3393-3396.

(39) Castelletto, V.; Hamley, I. W.; Stain, C.; Connon, C. SlowRelease RGD-Peptide Hydrogel Monoliths. Langmuir 2012, 28, 12575-12580.

(40) Martin, A. D.; Wojciechowski, J. P.; Bhadbhade, M. M.; Thordarson, P. A Capped Dipeptide Which Simultaneously Exhibits Gelation and Crystallization Behavior. Langmuir 2016, 32, 22452250.

(41) Viswanadhan, V. N.; Ghose, A. K.; Revankar, G. R.; Robins, R. K. Atomic Physicochemical Parameters for Three Dimensional Structure Directed Quantitative Structure-Activity Relationships. 4. Additional Parameters for Hydrophobic and Dispersive Interactions and Their Application for an Automated Superposition of Certain Naturally Occurring Nucleoside Antibiotics. J. Chem. Inf. Model. 1989, 29, 163-172.

(42) Garcia, A. M.; Iglesias, D.; Parisi, E.; Styan, K. E.; Waddington, L. J.; Deganutti, C.; De Zorzi, R.; Grassi, M.; Melchionna, M.; Vargiu, A. V.; Marchesan, S. Chirality Effects on Peptide Self-Assembly Unraveled from Molecules to Materials. Chem 2018, 4, 1862-1876.

(43) Amdursky, N.; Erez, Y.; Huppert, D. Molecular Rotors: What Lies Behind the High Sensitivity of the Thioflavin-T Fluorescent Marker. Acc. Chem. Res. 2012, 45, 1548-1557.

(44) Görbitz, C. H. Nanotube Formation by Hydrophobic Dipeptides. Chem. - Eur. J. 2001, 7, 5153-5159.

(45) Sawaya, M. R.; Sambashivan, S.; Nelson, R.; Ivanova, M. I.; Sievers, S. A.; Apostol, M. I.; Thompson, M. J.; Balbirnie, M.; Wiltzius, J. J.; McFarlane, H. T.; Madsen, A. O.; Riekel, C.; Eisenberg, D. Atomic Structures of Amyloid Cross-Beta Spines Reveal Varied Steric Zippers. Nature 2007, 447, 453-457.

(46) Cavallo, G.; Metrangolo, P.; Milani, R.; Pilati, T.; Priimagi, A.; Resnati, G.; Terraneo, G. The Halogen Bond. Chem. Rev. 2016, 116, 2478-2601.

(47) Sasselli, I. R.; Halling, P. J.; Ulijn, R. V.; Tuttle, T. Supramolecular Fibers in Gels Can Be at Thermodynamic Equilibrium: A Simple Packing Model Reveals Preferential Fibril Formation versus Crystallization. ACS Nano 2016, 10, 2661-2668.

(48) Hernández, B.; Pflüger, F.; Kruglik, S. G.; Ghomi, M. Characteristic Raman Lines of Phenylalanine Analyzed by a Multiconformational Approach. J. Raman Spectrosc. 2013, 44, 827833.

(49) Messina, M. T.; Metrangolo, P.; Navarrini, W.; Radice, S.; Resnati, G.; Zerbi, G. Infrared and Raman Analyses of the HalogenBonded Non-Covalent Adducts Formed by $\alpha, \omega$-Diiodoperfluoroalkanes with DABCO and Other Electron Donors. J. Mol. J. Mol. Struct. 2000, 524, 87-94.

(50) Li, H.; Lantz, R.; Du, D. Vibrational Approach to the Dynamics and Structure of Protein Amyloids. Molecules 2019, 24, 186.
(51) Wang, Q.; Wang, Y.; Lu, H. P. Revealing the Secondary Structural Changes of Amyloid $\beta$ Peptide by Probing the Spectral Fingerprint Characters. J. Raman Spectrosc. 2013, 44, 670-674.

(52) Lekprasert, B.; Korolkov, V.; Falamas, A.; Chis, V.; Roberts, C. J.; Tendler, S. J. B.; Notingher, I. Investigations of the Supramolecular Structure of Individual Diphenylalanine Nano- and Microtubes by Polarized Raman Microspectroscopy. Biomacromolecules 2012, 13, 2181-2187.

(53) Iglesias, D.; Melle-Franco, M.; Kurbasic, M.; Melchionna, M.; Abrami, M.; Grassi, M.; Prato, M.; Marchesan, S. Oxidized Nanocarbons-Tripeptide Supramolecular Hydrogels: Shape Matters! ACS Nano 2018, 12, 5530-5538.

(54) Pettersen, E. F.; Goddard, T. D.; Huang, C. C.; Couch, G. S.; Greenblatt, D. M.; Meng, E. C.; Ferrin, T. E. UCSF Chimera-A Visualization System for Exploratory Research and Analysis. J. Comput. Chem. 2004, 25, 1605-1612.

(55) Genheden, S.; Ryde, U. The MM/PBSA and MM/GBSA Methods to Estimate Ligand-Binding Affinities. Expert Opin. Drug Discovery 2015, 10, 449-461. 